\title{
Feature Displacement Interpolation
}

\author{
Mads Nielsen \\ 3D-Lab, School of Dentistry \\ University of Copenhagen \\ Copenhagen, Denmark
}

\author{
Per R. Andresen \\ Institute of Mathematical Modelling \\ Technical University of Denmark \\ Lyngby, Denmark
}

\begin{abstract}
Given a sparse set of feature matches, we want to compute an interpolated dense displacement map. The application may be stereo disparity computation, flow computation, or non-rigid medical registration. Also estimation of missing image data, may be phrased in this framework. Since the features often are very sparse, the interpolation model becomes crucial. We show that a maximum likelihood estimation based on the covariance properties (Kriging) show properties more expedient than methods such as Gaussian interpolation or Tikhonov regularizations, also including scale-selection. The computational complexities are identical. We apply the maximum likelihood interpolation to growth analysis of the mandibular bone. Here, the features used are the crest-lines of the object surface.
\end{abstract}

\section{Introduction}

Given images of an object and a deformed version of the object we wish to compute the displacement field. The overall strategy is to extract features [8], match these, and then interpolate the displacement field. Since feature extraction and matching is not ideal operations, only a very sparse set of reliable features may be extracted and matched. In our current application to 3D human bone-growth analysis, we use the crest-lines of the surface as features [12]. In this paper, we assume the features and matches are given, and examine the interpolation problem. Since the images are very large 3D images, we only consider direct methods, and not more computational heavy methods such as those based on functional minimisation [2, 9].

In the following we describe methods such as Gaussian interpolation [6], Tikhonov regularizations [13, 5], kriging [4], and an adaptive scheme, and describe their different properties. Finally, we apply the kriging to the growth analysis on synthetic 3D images of cubes, and the mandibular bone obtained from 3D CT scans of the same patient at different time instances [1].

\section{Displacement interpolation}

The values of a displacement field $f: \mathbb{R}^{D} \mapsto \mathbb{R}^{D}$ is provided by feature matches in a sparse set of points $\vec{x}=\left[x_{1}, x_{2}, \ldots, x_{n}\right]$ as $g(\vec{x})$. In the following, we keep $D=1$ for notational simplicity, whereas $D=3$ in the final computational examples. We want the following properties of the interpolation scheme:

- (i) Approximation criterion. $f$ must approximate the data values well since localisation of the features are assumed relatively precise.

- (ii) Regularity criterion. In regions of missing features a regular solution must be created. In general, we will require smoothness $\left(C^{\infty}\right)$, but only $C^{1}$ and visual regularity (no extra structure introduced).

- (iii) Asymptotic behaviour. The data must be able of shadowing each other. That is, in a given direction only the nearest data must be weighted.

- (iv) Maximum principle. The solution must not extent the solution to values larger than the largest data value or smaller that the smallest data value.

Among the above mentioned interpolation methods, we analyse for these criteria.

\subsection{Tikhonov regularization or thin-plate splines}

A displacement field can be reconstructed as a Maximum A Posteriori (MAP) estimate given noise estimates and a prior of displacement fields. Especially, when the prior is an uncorrelated Gaussian in the displacement gradient magnitude, we obtain Tikhonov regularization [10]:

$$
f=\arg \min E[f],
$$

where

$$
E[f]=\sum_{i}\left(g\left(x_{i}\right)-f\left(x_{i}\right)\right)^{2}+\lambda \int_{\mathbb{R}}\left(\partial_{x} f\right)^{2} d x
$$


The solution may be obtained as [11]:

$$
f(x)=\frac{w(x, \vec{x}) \cdot g(\vec{x})}{w(x, \vec{x}) \cdot \overrightarrow{1}}
$$

where $g(\vec{x})$ is a vector containing the data values in the data points $x$ arranged in the vector $\vec{x}, \overrightarrow{1}$ is a vector containing 1's, and $w(x, \vec{x})$ is a vector containing values $w_{i}(x)=e^{-\frac{\left|x-x_{i}\right|}{\lambda}}$. This is also known as the unbiased estimation of Thin-plate splines [3]. The essential part here is the shape of the filter $\left(e^{-|x|}\right)$ and that this may be perceived as a standard filtering of the unevenly distributed data points with a re-normalisation so that the total filter weight becomes unity. This method does not comply with the approximation criterion and the asymptotic behaviour, but does fulfil the maximum principle. The solutions are not in a mathematical sense smooth, but only $C^{1}$, which is sufficient for our applications..

\subsection{Gaussian interpolation}

Knutson and Westin [6] proposed a similar filtering, but based on Gaussian filters: $w_{i}(x)=e^{-\frac{\left(x-x_{i}\right)^{2}}{2 \lambda^{2}}}$. This filter shows different properties than the Tikhonov filter, especially far from data points, which is interesting in the case of very sparse data. It has the required asymptotic behaviour. A theoretical difference is that Tikhonov regularizations yields $C^{1}$ functions while the Gaussian filter yields $C^{\infty}$ functions.

The parameter $\lambda$ yields in both methods a trade off between over-smoothing in regions where many data are given (violation of (i)) and making smooth solutions in areas where only few data are given (property (ii)).

\subsection{Adaptive Gaussian filtering}

A solution to the violations in the normalised filterings may be a local adaption of the scale parameter to the distance of the nearest features: $\lambda(x)=$

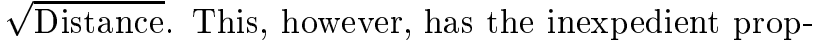
erty that data points cannot "shadow" each other. That is, far from a step edge, the solution will take an intermediate value, thus we have violated property (iii). Furthermore, in vast regions, structure may be introduced (violation of (ii)). Other principles of selecting the scale may exhibit different properties.

\subsection{Kriging or Gaussian regression}

The basic problem of the normalised filtering method is that the belief in the smoothness of the solution and the belief in the accuracy of the data values are merged into one smoothness parameter $\lambda$. We can phrase the statistical inference problem a little differently and then separate these two parameters, thus yielding kriging [4]:
Assume, instead of a prior on the derivative, that the covariance function $C\left(x, x^{\prime}\right)$ is known. The covariance function expresses the covariance of the data values in two points $x$ and $x^{\prime}$. Typically the closer points are, the more correlated their data values are assumed to be. An interesting aspect is that if this covariance defines a distribution of functions, and if $C\left(x, x^{\prime}\right)=e^{-\left(\frac{\left|x-x^{\prime}\right|}{\lambda}\right)^{\alpha}}$, some well-known function classes appear with probability 1 , for different choices of $\alpha: \alpha=0$ yields white noise, $\alpha \in] 0 ; 2[$ yields fractional Brownian motions with $\alpha=1$ as the classical Brownian motion [7], while $\alpha=2$ (the Gaussian) yields $C^{\infty}$ functions.

Given the covariance function $C\left(x, x^{\prime}\right)$ and an expression of the belief in data as the assumed variance of data values $r^{2}$, we can make a maximum likelihood estimation of $f(x)$ as [14]

$$
f(x)=\frac{w(x, \vec{x}) Q^{-1} g(\vec{x})}{w(x, \vec{x}) Q^{-1} \overrightarrow{1}}
$$

where $w(x, \vec{x})$ is a vector containing $w_{i}=C\left(x, x_{i}\right)$, and $Q$ is a matrix containing $Q_{i j}=C\left(x_{1}, x_{2}\right)+r^{2} \delta_{i j}$. The intuitive interpretation of the introduction of $Q^{-1}$ is that, prior to the regularizations based on the covariance function, an inverse filtering is performed to make the samples uncorrelated. In terms of scale-space we might say that we have data given at some scale $\lambda$. To interpolate we first perform a deblurring to scale zero, interpolate, and then blur back to the current scale. The solution has the same regularity properties as the corresponding normalised filtering ( $\alpha=1$, thin-plate splines are $C^{1}$ and $\alpha=2$, normalised Gaussian filtering is $C^{\infty}$ ). Varying the parameter $r$ yields different properties of the solutions, and in the limit $r \mapsto \infty$, we are back to normalised filtering.

\section{Interpolation properties}

The properties of the different interpolation models are noted in the figure captions below. Below we give a table indicating properties. Notice that all methods except the adaptive scale method may be formulated as Kriging. If $\alpha=1$ and $r^{2}=\infty$ Kriging yields Tikhonov regularization, while $\alpha=2$ and $r^{2}=\infty$ yields Gaussian interpolation.

\begin{tabular}{|l|c|c|c|c|}
\hline Method & (i) & (ii) & (iii) & (iv) \\
\hline$\alpha=1, r^{2}=0$ & + & $C^{1}$ & + & + \\
\hline$\alpha=1, r^{2}=1$ & - & $C^{1}$ & - & + \\
\hline$\alpha=1, r^{2}=\infty$ & - & $C^{1}$ & - & + \\
\hline$\alpha=2, r^{2}=0$ & + & $C^{\infty}$ & - & - \\
\hline$\alpha=2, r^{2}=1$ & - & $C^{\infty}$ & - & - \\
\hline$\alpha=2, r^{2}=\infty$ & - & $C^{\infty}$ & + & + \\
\hline Adaptive scale & + & $C^{1}$ & - & + \\
\hline
\end{tabular}




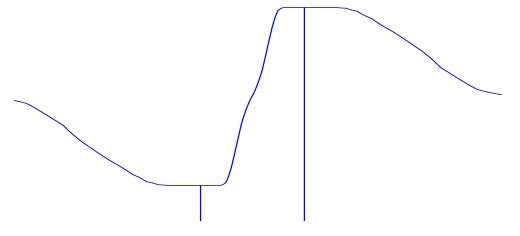

Figure 1: Adaptive filtering, $\alpha=2$. This violates the principle of asymptotic behaviour, only the closest value should be used. Furthermore notice the bump in the middle; extra structure is introduced thus violating the regularity criterion.

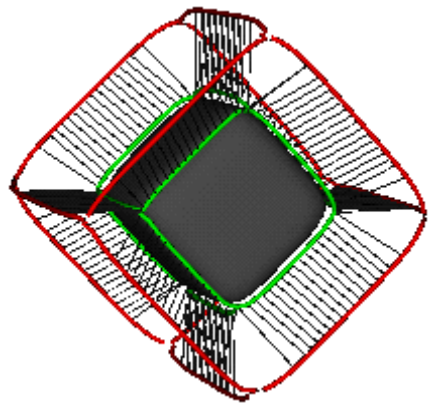

Figure 2: Crest-lines and their matchings on two cubes of same orientation and position, but with a relative size change of $1 / 3$. Results are obtained automatically. Notice the erroneous matching so that only the central part of the crest-lines at the larger cube is used. This is made deliberately so as to expose the interpolation properties.

\section{Summary}

We are given very reliable, but also very sparse feature matches. Based upon this situation we have formulated 4 criteria for a displacement interpolation. Among standard regularization schemes they cannot be fulfilled simultaneously. Using a formulation of the interpolation problem normally applied in geostatistics, Kriging, we may fulfil all criteria simultaneously, having only one free scale parameter. This has been applied to 3D growth analysis based upon crest-line matches.

\section{References}

[1] P. R. Andresen, M. Nielsen, S. Kreiborg. $4 D$ shape-preserving modelling of bone growth To appear in Proc. of Medical Image Computing and Computer-Assisted Interventions, Cambridge MA, October 11-13, 1998.

[2] A. Blake and A. Zisserman. Visual Reconstruction. MIT Press, Cambridge, Mass., 1987.

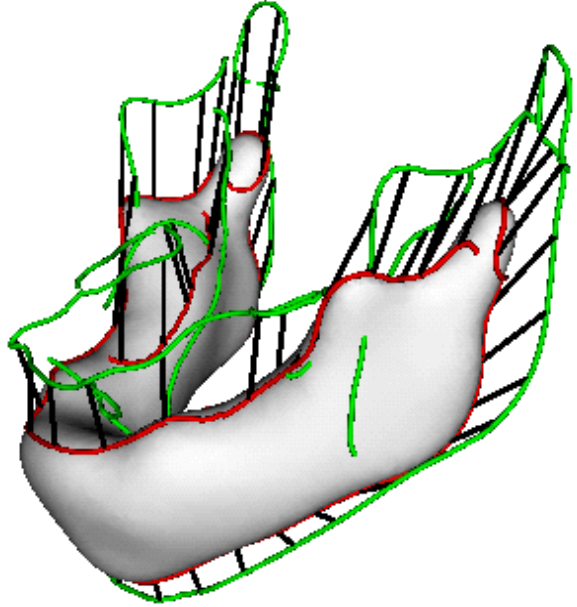

Figure 8: Crest line matches on mandibular bone of the same patient scanned at age 21 months and 7 years.
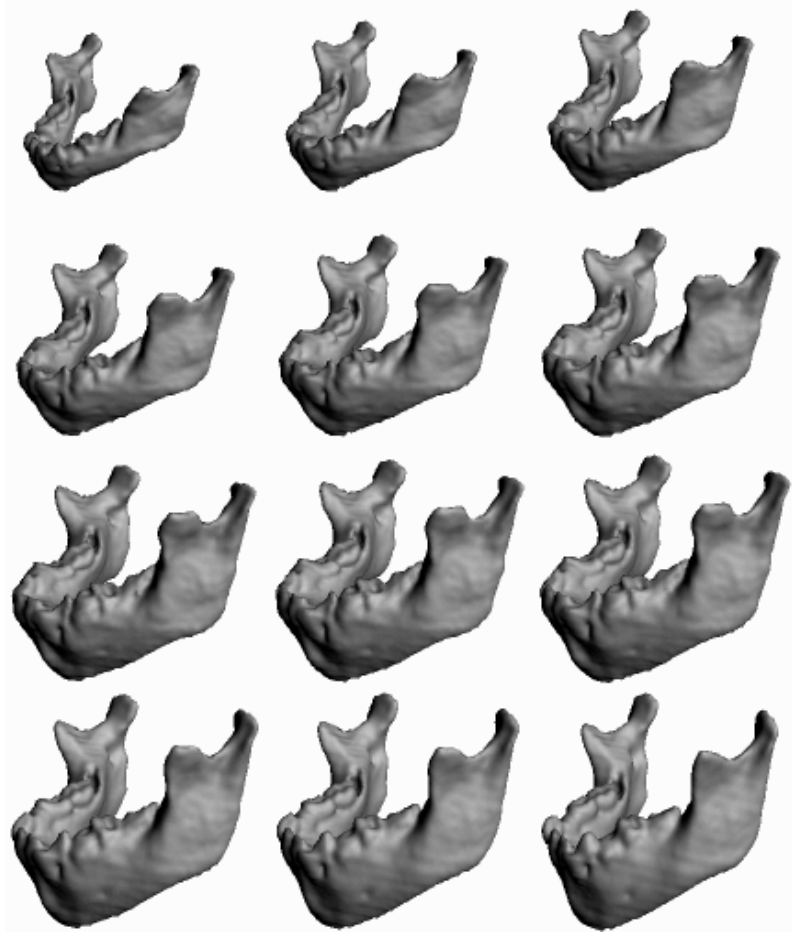

Figure 9: Interpolated growth of the mandible based on $3 \mathrm{CT}$ scans at age 9 months, 21 months, and 7 years. Spatial interpolation based on the parameter free adaptive scale method, temporal interpolation based upon second order polynomialb model. 


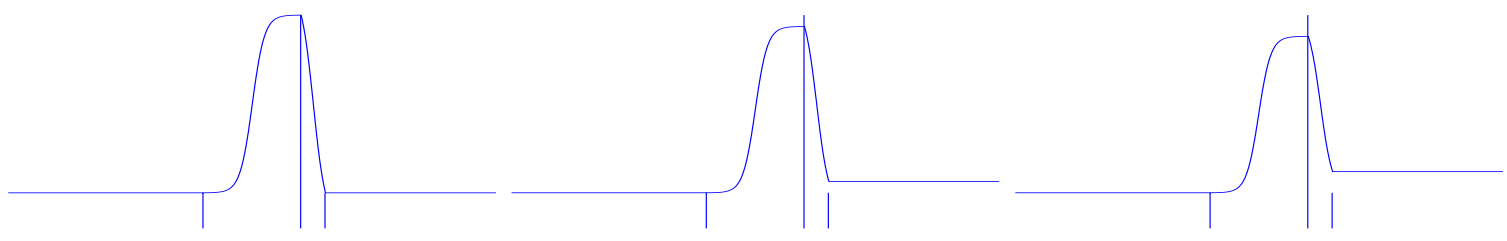

Figure 3: Kriging, $\alpha=1, \lambda=50$, from left to right $r^{2}=0, r^{2}=1, r^{2} \approx \infty$. The vertical lines corresponds to data points with values corresponding to the height of the lines. The left figure is the only of all the interpolation techniques in this paper that satisfy all 4 criteria. When $r^{2}>0$ the approximation and asymptotic behaviour are violated.



Figure 4: Kriging, $\alpha=1, \lambda=5$, from left to right $r^{2}=0, r^{2}=1, r^{2} \approx \infty$. This figure is identical to the above with the exception of a smaller scale, which makes the solution go towards a step function.

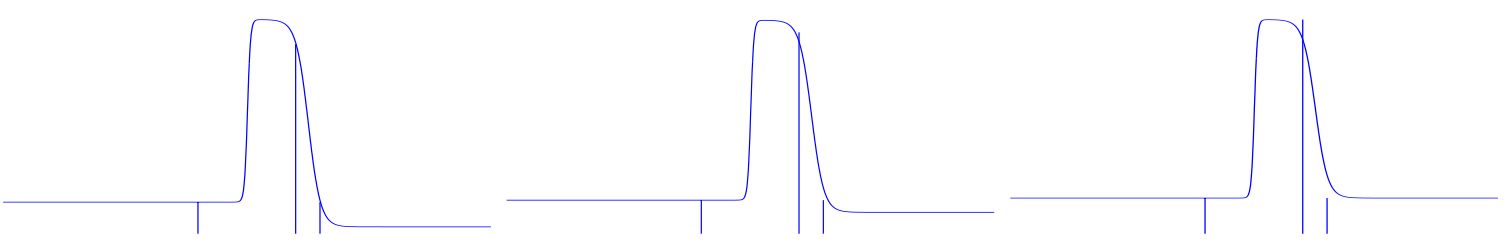

Figure 5: Kriging, $\alpha=2, \lambda=50$, from left to right $r^{2}=0, r^{2}=1, r^{2} \approx \infty$. Here, the left violates the maximum principle and the asymptotic criterion, while the right violates the approximation principle. The middle violates all three, but to a smaller extent.
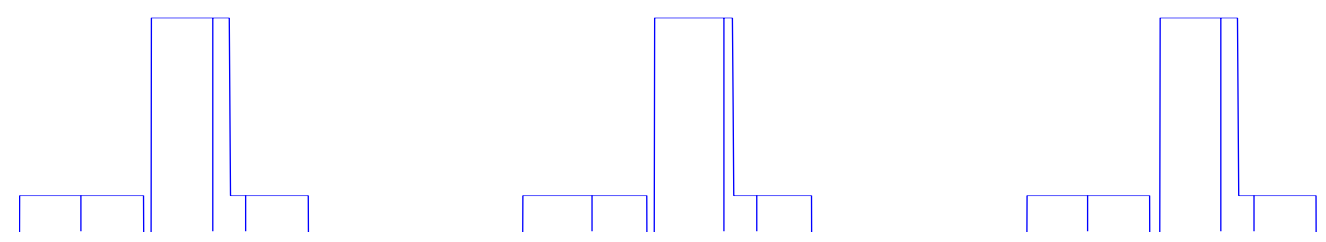

Figure 6: Kriging, $\alpha=2, \lambda=5$, from left to right $r^{2}=0, r^{2}=1, r^{2} \approx \infty$. The same as the above, but width smaller scale. Here we run into numerical problems and in some points no solution is given. Furthermore, the solutions are closer to a step function, making the above criteria violations smaller. 

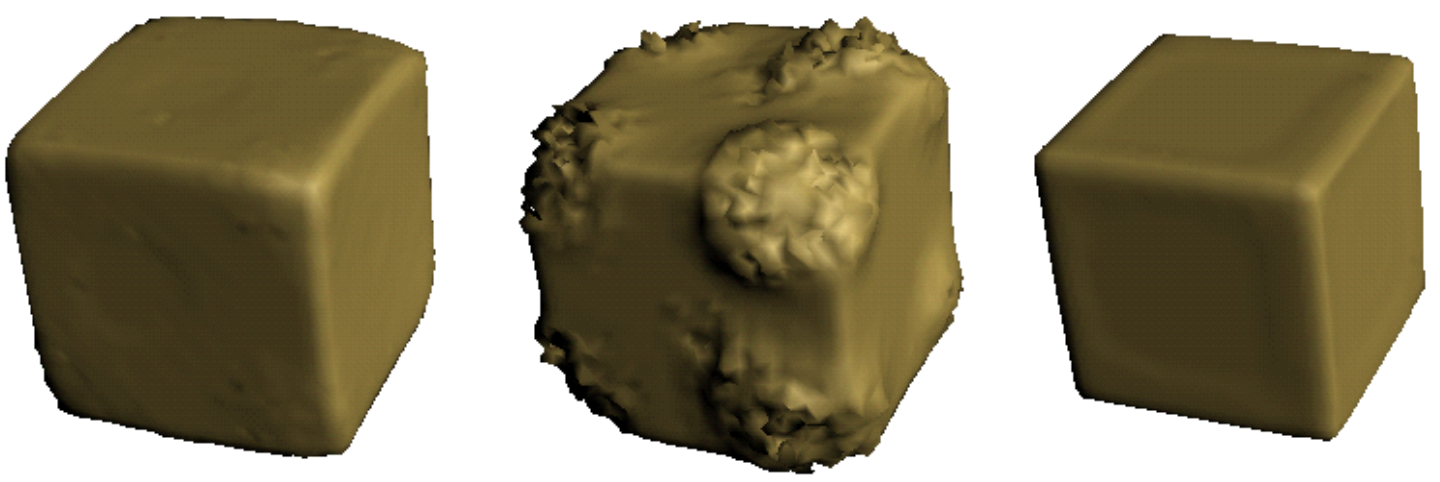

Figure 7: Reconstruction of the smaller cube at the size of the larger cube. Displacements are computed on the basis of crest-lines on the two cubes, matching and interpolation using $\alpha=1, \sigma=2, r^{2}=10^{-3}$ (left), $\alpha=2, \sigma=2, r^{2}=10^{-3}$ (middle), $\alpha=1$, $\sigma=2, r^{2} \approx \infty$ (right). The leftmost corresponds to the only interpolation not violating any criteria. The central does not satisfy the maximum principle, and the asymptotic behaviour, while the rightmost does not satisfy approximation criterion, hence the resulting cube is too small.

[3] F. L. Bookstein. Morphometric Tools for Landmark Data. Cambridge University Press, 1991.

[4] I. Clark. Practical Geostatistics. Elsevier Applied Science, 1979.

[5] W. E. L. Grimson. From Images to Surfaces. MIT Press, Cambridge MA, 1981.

[6] H. Knutsson and C.F. Westin. Normalized and differential convolution. In CVPR93, pages 515$523,1993$.

[7] B. B. Mandelbrot and J. W. van Ness. Fractional Brownian motions, fractional noises, and applications. SIAM Review, 10(4):422-437, October 1968.

[8] D. Marr. Vision. W. H. Freeman \& Co., 1882.

[9] D. Mumford and J. Shah. Boundary detection by minimizing functionals. In Proc. IEEE Conf. on Computer Vision and Pattern Recognition, San Francisco, 1985.

[10] Mads Nielsen. From Paradigm to Algorithms in Computer Vision. PhD thesis, DIKU, Datalogisk Institut ved Københavns Universitet, Copenhagen, Denmark, April 1995. DIKU-95-8.

[11] Mads Nielsen, Luc Florack, and Rachid Deriche. Regularization, scale space, and edge detection filters. Journal on Mathematical Imaging and Vision, 7:291-307, 1997.
[12] J.-P. Thirion and A. Gourdon. Computing the differential characteristics of isodensity surfaces. Computer Vision, Graphics, and Image Processing: Image Understanding, 61(2):109-202, March 1995.

[13] A. N. Tikhonov and V. Y. Arsenin. Solution of IllPosed Problems. Winston and Wiley, Washington DC, 1977.

[14] C. K. I. Williams and C. E. Rasmussen. Gaussian processes for regression. Advances in Neural Information Processing Systems, 8, 1996. 